\title{
Usefulness of neutrophil-to-lymphocyte ratio and platelet-to-lymphocyte ratio in hormone- receptor-negative breast cancer
}

This article was published in the following Dove Press journal:

OncoTargets and Therapy

27 July 2016

Number of times this article has been viewed

\author{
Chao Liu ${ }^{1,2}$ \\ Zhou Huang' \\ Qiusheng Wang' \\ Bing Sun' \\ Lijuan Ding' \\ Xiangying Meng' \\ Shikai $\mathrm{Wu}^{1,2}$ \\ 'Department of Radiation Oncology, \\ Affiliated Hospital of Academy \\ of Military Medical Sciences, \\ ${ }^{2}$ Department of Radiation Oncology, \\ 307 Hospital of PLA, 307 Clinical \\ College, Anhui Medical University, \\ Beijing, People's Republic of China
}

Purpose: We aimed to investigate the relationship between pretreatment neutrophil-to-lymphocyte ratio (NLR)/platelet-to-lymphocyte ratio (PLR) and the estimation of hormone-receptor-negative (HR-) breast cancer patients' survival in a Chinese cohort.

Patients and methods: Of 434 consecutive HR- nonmetastatic breast cancer patients treated between 2004 and 2010 in the Affiliated Hospital of Academy of Military Medical Sciences, 318 eligible cases with complete data were included in the present study. Kaplan-Meier analysis was performed to determine the overall survival (OS) and disease-free survival (DFS). Univariate and multivariate Cox proportional hazards models were used to test the usefulness of NLR and PLR.

Results: Univariate analysis indicated that both elevated NLR and PLR (both $P<0.001$ ) were associated with poor OS. The utility of NLR remained in the multivariate analysis $(P<0.001)$, but not PLR $(P=0.104)$. The analysis results for DFS were almost the same as OS. Subgroup analysis revealed a significant association between increased NLR and PLR $(P<0.001$ and $P=0.011$ ) and poor survival in triple-negative breast cancer. However, for human epidermal growth factor receptor 2-positive breast cancer, only NLR was significantly associated with OS in the multivariate analysis $(P=0.001)$.

Conclusion: The present study indicates that both increased NLR and PLR are associated with poor survival in HR- breast cancer patients. Meanwhile, NLR is independently correlated with OS and DFS, but PLR is not.

Keywords: inflammatory markers, breast cancer, survival

\section{Introduction}

Breast cancer is the most frequently diagnosed cancer in females worldwide, including within the People's Republic of China. ${ }^{1}$ Newly diagnosed Chinese patients account for $12.2 \%$ of those all over the world. In spite of the falling mortality, the number of deaths is still sufficiently numerous each year. ${ }^{2}$ Hormone-receptor-negative (HR-) breast cancer, including human epidermal growth factor receptor 2-positive (HER2+, nonluminal B) breast cancer, and triple-negative breast cancer (TNBC), has high recurrence and distant metastasis rates and the worst prognosis among subtypes. ${ }^{3}$ Consequently, it is highly significant to predict the prognosis of these patients, which influences clinicians' treatment decision. Currently, the main prognostic factors for HR- breast cancer are age, tumor size, grade, nodal status, and HER2 status. ${ }^{4}$ Although the usefulness of some new predictors (such as gene profiling, circulating tumor cells, and tumor T-cell infiltration) has been investigated, their clinical use is limited because of the uncertain role and high costs..$^{5-7}$
Department of Radiation Oncology, Affiliated Hospital of Academy of Military Medical Sciences, 8 East Road, Fengtai District, Beijing 10007I,

People's Republic of China

Tel +861066946830

Fax +86106694 7196

Email skywu4923@sina.com 
Recently, there is growing evidence that systemic inflammation is associated with the prognosis of various cancers, including breast cancer. ${ }^{8,9}$ Systemic inflammatory response could be expressed by some biochemical or hematological markers, such as raised C-reactive protein levels, hypoalbuminemia, and the elevation of white cell, neutrophil, and platelet counts. A combination of these factors was used to derive prognostic scores for inflammation, such as the Glasgow prognostic score, the neutrophil-to-lymphocyte ratio (NLR), and the platelet-to-lymphocyte ratio (PLR). ${ }^{10}$

To date, the unfavorable role of elevated NLR and PLR was deeply investigated in gastrointestinal tumors and non-smallcell lung cancers. ${ }^{11-16}$ However, the usefulness of NLR and PLR in breast cancer patients is not well studied, especially in $\mathrm{HR}$ - breast cancer patients on account of its low incidence. , 17-22 $^{-12}$ Three studies suggested that increased NLR is associated with poor prognosis in TNBC, ${ }^{8,17,19}$ but another two did not. ${ }^{21,22}$ The correlation between increased PLR and the prognosis of TNBC is conflicting. ${ }^{8,19,20}$ For HER2+ breast cancer, the utility of NLR and PLR is vague..$^{8,19,21}$ In addition to these conflicting results, the scales of published studies for HR-breast cancer are small. Therefore, we aimed to further investigate the usefulness of pretreatment NLR and PLR in a comparatively large cohort of Chinese HR- breast cancer patients.

\section{Patients and methods}

We retrospectively evaluated $\mathrm{HR}$ - breast cancer patients treated between June 2004 and June 2010 in Affiliated Hospital of Academy of Military Medical Sciences. Among 434 HRbreast cancer patients, a total of 318 eligible patients were included. The inclusion criteria were as follows: histologically confirmed HR-nonmetastatic breast cancer, with pretreatment data of differential blood counts, and no history of inflammatory and immune disease, diabetes, hypertension, metabolic syndrome, coronary artery disease, renal disease, and hematological disease. Patients with metastatic and inflammatory breast tumors and infectious diseases were excluded.

Estrogen receptor (ER) negative and progesterone receptor $(\mathrm{PR})$ negative were defined as $<10 \%$ of positive invasive tumor nuclei by immunohistochemistry test. HER2 status was negative when the immunohistochemistry result was 0 and positive when the result was $3+$ or when fluorescence in situ hybridization confirmed positivity for $1+$ and $2+$ patients. Blood count results from pretreatment (surgery and neoadjuvant chemotherapy) tests were obtained and confirmed by online records. Clinical and pathological characteristics included age, tumor size, lymph node involvement, tumor stage (American Joint Committee on Cancer [AJCC]-6 criteria), grade, and ER, PR, and HER2 status. The treatments for HR- breast cancer included surgery (breast-conserving surgery and mastectomy), chemotherapy (adjuvant and neoadjuvant chemotherapy), targeted therapy (trastuzumab and lapatinib), and adjuvant radiotherapy.

The NLR/PLR was calculated by dividing the neutrophil/ platelet count by the lymphocyte count. The primary end points were overall survival (OS) and disease-free survival (DFS). OS was defined as the interval between the date of histological diagnosis and the time of death of any cause. DFS was calculated from the time of surgery to the time of first relapse or death. The follow-up time was calculated from the time of diagnosis to the time of death or end up. Follow-up was censored when the patient was dead or at the end time of October 20,2015. Nineteen patients were lost to follow-up at the end time. This study was approved by the ethical committee of the Affiliated Hospital of Academy of Military Medical Sciences. The ethical committee of the Affiliated Hospital of Academy of Military Medical Sciences did not require written informed consent be obtained from all patients, as this was a retrospective study, and all data was anonymous.

\section{Statistical analysis}

The optimal cutoff values for NLR and PLR were determined by employing receiver operating curve (ROC) analysis to discriminate high and low OS. The association between the NLR/PLR and pathological characteristics was evaluated by nonparametric tests. Kaplan-Meier method and log-rank test were used to study the primary end points. Univariate and multivariate Cox proportional hazards models were performed to obtain prognostic factors and corresponding hazard ratios (HRs) for different factors with $95 \%$ confidence intervals (CIs). To investigate the prognostic effect of NLR and PLR in different subtypes, HR- breast cancer was also divided into HER2+ breast cancer and TNBC. The multivariate analysis included age, tumor size, lymph node status, tumor grade, HER2 status, NLR, and PLR. All tests were two sided, and $P$-value $<0.05$ was considered statistically significant. Statistical Package for Social Sciences Version 20.0 (IBM Corporation, Armonk, NY, USA) was used to analyze the statistics.

\section{Results}

Among 318 eligible patients, 157 (49.4\%) and 161 (50.6\%) patients were classified into HER2+ breast cancer patients and TNBC patients, respectively. The baseline characteristics of 
Table I Baseline characteristics

\begin{tabular}{|c|c|}
\hline Characteristics & $\begin{array}{l}\text { Number of } \\
\text { patients (\%) }\end{array}$ \\
\hline \multicolumn{2}{|l|}{ Age (years) } \\
\hline$\leq 35$ & $55(17.3)$ \\
\hline$>35$ & $263(82.7)$ \\
\hline \multicolumn{2}{|l|}{ Tumor stage } \\
\hline $\mathrm{TI}$ & $67(21.1)$ \\
\hline $\mathrm{T} 2$ & $178(56)$ \\
\hline $\mathrm{T} 3$ & $61(19.2)$ \\
\hline $\mathrm{T} 4$ & $10(3.1)$ \\
\hline Unknown & $2(0.6)$ \\
\hline \multicolumn{2}{|l|}{ Lymph node status } \\
\hline No & $127(39.9)$ \\
\hline $\mathrm{NI}$ & $87(27.4)$ \\
\hline N2 & $47(14.8)$ \\
\hline N3 & $55(17.3)$ \\
\hline Unknown & $2(0.6)$ \\
\hline \multicolumn{2}{|l|}{ AJCC stage } \\
\hline I & $47(14.8)$ \\
\hline II & I5I (47.5) \\
\hline III & II 8 (37.I) \\
\hline Unknown & $2(0.6)$ \\
\hline \multicolumn{2}{|l|}{ Tumor grade } \\
\hline GI & $29(9.1)$ \\
\hline G2 & $170(53.5)$ \\
\hline G3 & $107(33.6)$ \\
\hline Unknown & $12(3.8)$ \\
\hline \multicolumn{2}{|l|}{ Surgery } \\
\hline $\mathrm{BCS}$ & $46(14.5)$ \\
\hline Mastectomy & $27 \mid(85.2)$ \\
\hline Unknown & $\mathrm{I}(0.3)$ \\
\hline \multicolumn{2}{|l|}{ Chemotherapy } \\
\hline NAC & $56(17.6)$ \\
\hline$A C$ & $259(81.5)$ \\
\hline None & $3(0.9)$ \\
\hline \multicolumn{2}{|c|}{ Adjuvant radiotherapy } \\
\hline Yes & $193(60.7)$ \\
\hline None & $122(38.4)$ \\
\hline Unknown & $3(0.9)$ \\
\hline \multicolumn{2}{|l|}{ Targeted therapy } \\
\hline Yes & $76(23.9)$ \\
\hline None & 235 (73.9) \\
\hline Unknown & $7(2.2)$ \\
\hline \multicolumn{2}{|l|}{ HER2 status } \\
\hline Positive & $157(49.4)$ \\
\hline Negative & $161(50.6)$ \\
\hline \multicolumn{2}{|l|}{ NLR } \\
\hline$<3$ & $195(6 \mid .3)$ \\
\hline$\geq 3$ & $123(38.7)$ \\
\hline \multicolumn{2}{|l|}{ PLR } \\
\hline$<147$ & $146(45.9)$ \\
\hline$\geq 147$ & $172(54.1)$ \\
\hline
\end{tabular}

Abbreviations: AJCC, American Joint Committee on Cancer; BCS, breastconserving surgery; NAC, neoadjuvant chemotherapy; AC, adjuvant chemotherapy; HER2, human epidermal growth factor receptor 2; NLR, neutrophil-to-lymphocyte ratio; PLR, platelet-to-lymphocyte ratio. all patients are shown in Table 1 . The median age at the time of diagnosis was 45 years (range 19-71 years). The median follow-up time was 58.1 months (range 5.9-136.1 months). The median OS and DFS were 63.5 months and 21.2 months, respectively.

The mean neutrophil, lymphocyte, and platelet counts were $3.8 \pm 1.2,1.5 \pm 0.5$, and $226.4 \pm 60.5$, respectively. The mean NLR and PLR were $2.8 \pm 1.3$ and $164.0 \pm 68.9$, respectively. ROC analysis revealed the optimal cutoff values of NLR and PLR as 3.0 and 147, respectively. The values of area under the curve were 0.70 and 0.67 for NLR (Figure 1) and PLR (Figure 2), respectively. The number of patients divided by high NLR/PLR and low NLR/PLR ratio is shown in Table 1. Nonparametric tests indicated that high NLR was associated with tumor size, lymph node status, AJCC stage, and surgery type. An increasing PLR significantly correlated with tumor size, lymph node status, AJCC stage, and HER2 status.

Among 318 patients, 283 (89.0\%) and 278 (87.4\%) developed recurrence disease and distant metastases at the end time, respectively. In all, 234 (73.6\%) patients died for any cause. OS and DFS of different groups divided by cutoff values of NLR and PLR are shown in Figures 3-6, respectively. In high NLR ( $\geq 3$ ) and PLR ( $\geq 147$ ) groups, the estimated median OSs were 42.3 months and 51.5 months, respectively. In the contrary, in low NLR and PLR groups, the estimated median OSs were 78.2 months and 79.3 months, respectively.

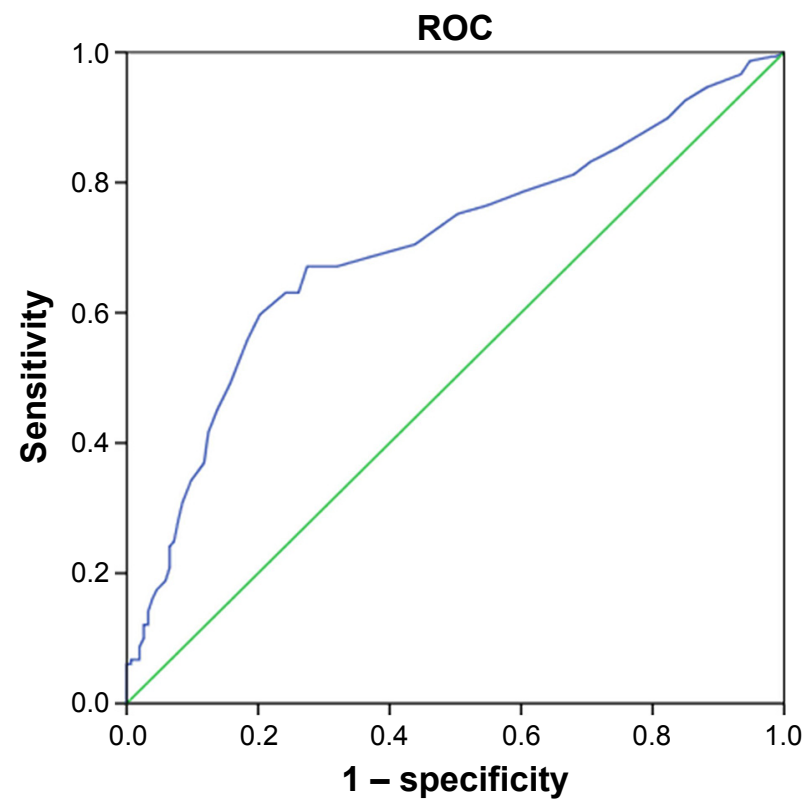

Figure I ROC analysis to discriminate high and low OS by NLR.

Note: The value of area under the curve is 0.70 .

Abbreviations: ROC, receiver operating curve; OS, overall survival; NLR, neutrophil-to-lymphocyte ratio. 


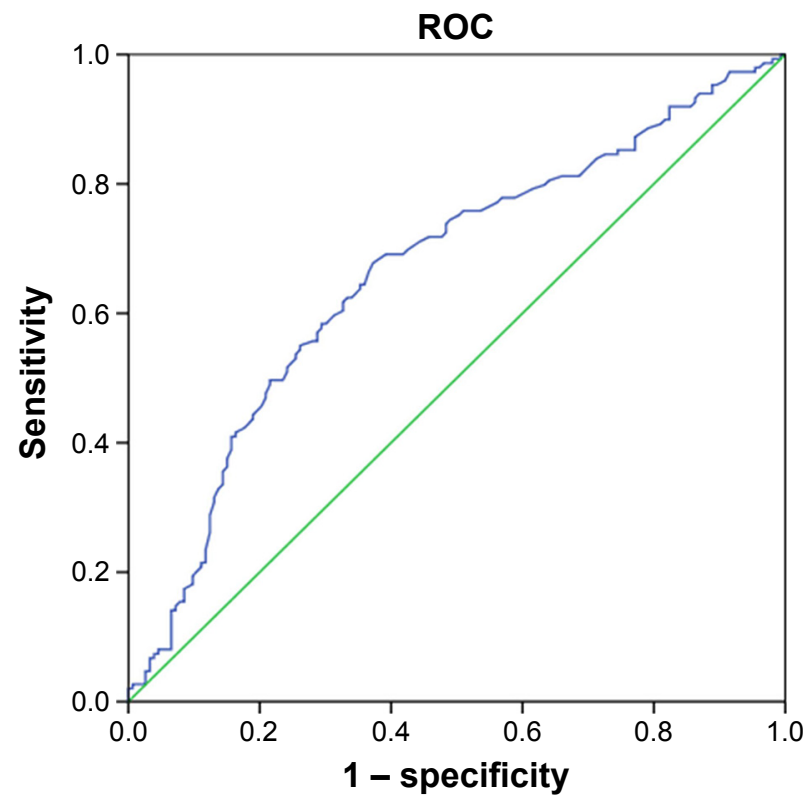

Figure 2 ROC analysis to discriminate high and low OS by PLR. Note: The value of area under the curve is 0.67 .

Abbreviations: ROC, receiver operating curve; OS, overall survival; PLR, plateletto-lymphocyte ratio.

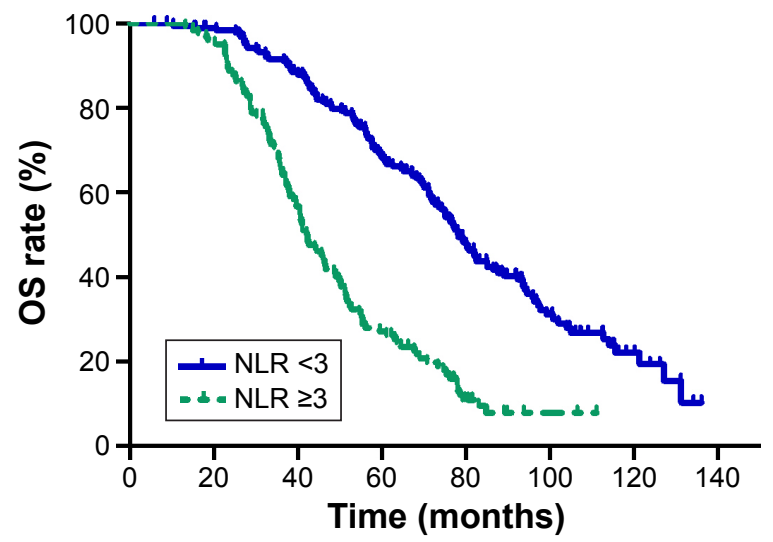

Figure 3 OS rate by low and high NLR $(P<0.001)$.

Abbreviations: OS, overall survival; NLR, neutrophil-to-lymphocyte ratio.

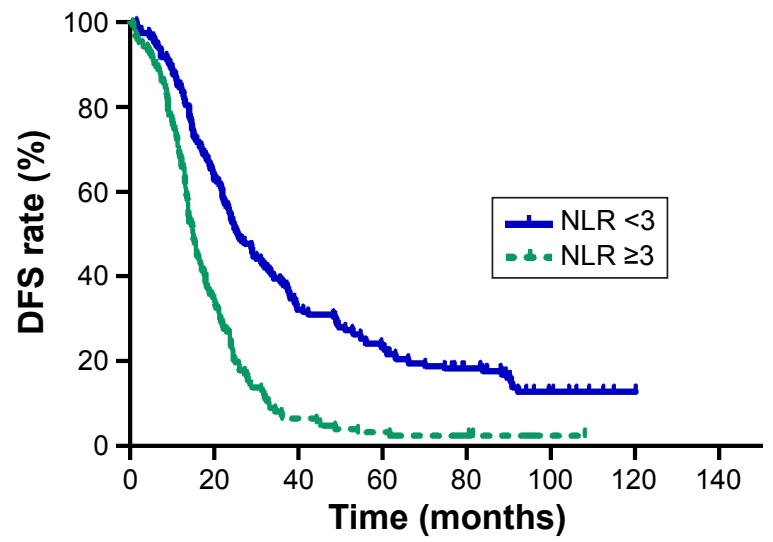

Figure 4 DFS rate by low and high NLR $(P<0.001)$.

Abbreviations: DFS, disease-free survival; NLR, neutrophil-to-lymphocyte ratio.

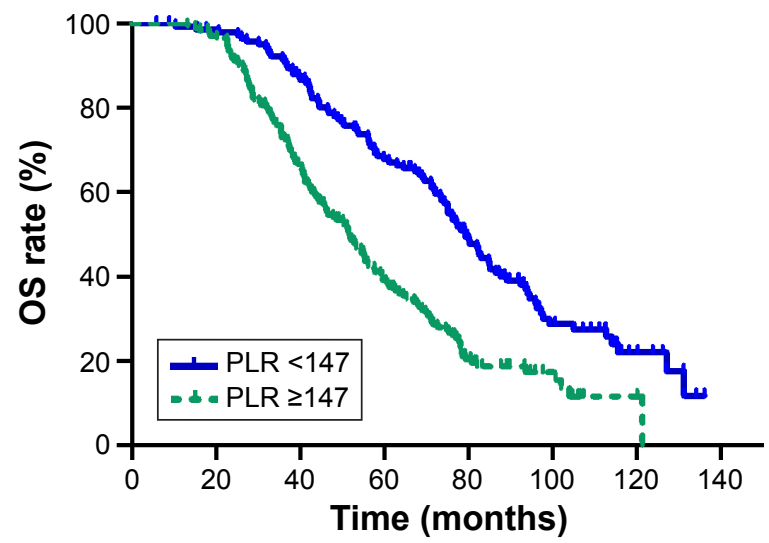

Figure 5 OS rate by low and high PLR $(P<0.001)$.

Abbreviations: OS, overall survival; PLR, platelet-to-lymphocyte ratio.

For OS, univariable analysis showed a significant association of high NLR and PLR with poor survival (Table 2). The predictive value of elevated NLR remained in the multivariable analysis (HR: $2.334,95 \%$ CI: $1.712-3.182$, $P<0.001$; Table 2), but not PLR (HR: $1.302,95 \%$ CI: $0.947-1.789, P=0.104$; Table 2). In addition, high tumor stage, grade, and lymph node involvement were recognized as indicators of poor survival in the multivariable analysis. High NLR and PLR also suggested poor outcome of patients' DFS in the univariable analysis. Meanwhile, the predictive value of high PLR was not found in the multivariable analysis, but NLR was found (Table 2). Furthermore, increased tumor size and high tumor grade were independent indicators for patients' DFS.

Subgroup analysis for different breast cancer subtypes revealed that elevated NLR (HR: $2.546,95 \%$ CI: $1.627-3.984$, $P<0.001$; Table 3) and PLR (HR: 1.709, 95\% CI: 1.130-2.585, $P=0.011$; Table 3) were both independent prognostic factors for OS of TNBC patients. However, PLR did not retain

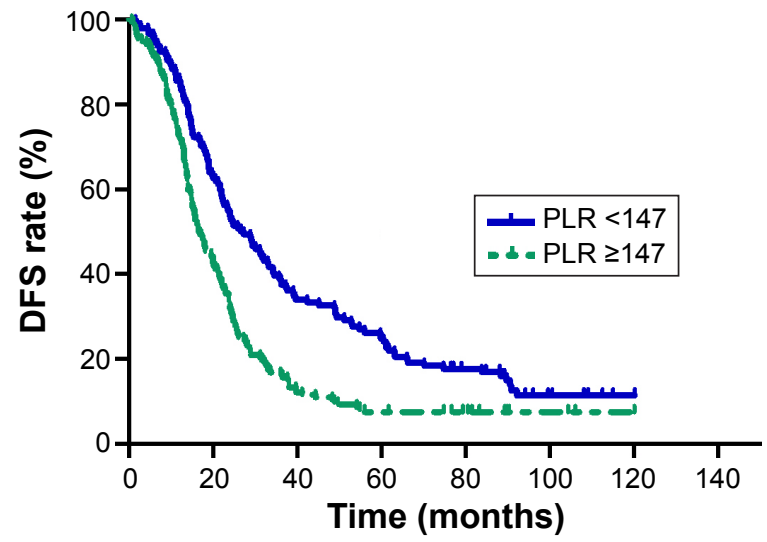

Figure 6 DFS rate by low and high PLR $(P<0.001)$.

Abbreviations: DFS, disease-free survival; PLR, platelet-to-lymphocyte ratio. 
Table 2 Association between clinicopathologic parameters and OS/DFS in $318 \mathrm{HR}$ - breast cancer patients

\begin{tabular}{|c|c|c|c|c|c|c|c|c|}
\hline \multirow[t]{3}{*}{ Variables } & \multicolumn{4}{|l|}{ OS } & \multicolumn{4}{|l|}{ DFS } \\
\hline & \multicolumn{2}{|l|}{ Univariate analysis } & \multicolumn{2}{|c|}{ Multivariate analysis } & \multicolumn{2}{|l|}{ Univariate analysis } & \multicolumn{2}{|c|}{ Multivariate analysis } \\
\hline & HR (95\% Cl) & $P$-value & HR $(95 \% \mathrm{Cl})$ & $P$-value & HR $(95 \% \mathrm{Cl})$ & $P$-value & HR $(95 \% \mathrm{Cl})$ & $P$-value \\
\hline \multicolumn{9}{|l|}{ Age (years) } \\
\hline$\leq 35$ vs $>35$ & $0.834(0.062-1.157)$ & 0.277 & $0.808(0.575-1.136)$ & 0.220 & $0.805(0.596-1.086)$ & 0.155 & $0.756(0.553-1.033)$ & 0.079 \\
\hline \multicolumn{9}{|l|}{ Tumor size } \\
\hline$<2$ vs $\geq 2$ & $2.628(1.828-3.776)$ & $<0.001$ & $1.863(1.264-2.747)$ & 0.002 & $2.186(1.615-2.959)$ & $<0.001$ & 1.607 (I.I62-2.222) & 0.004 \\
\hline \multicolumn{9}{|c|}{ Lymph node involvement } \\
\hline No vs yes & 2.048 (1.555-2.697) & $<0.001$ & 1.364 (1.009-1.843) & 0.044 & I.77I (I.389-2.559) & $<0.001$ & $1.263(0.968-1.147)$ & 0.085 \\
\hline \multicolumn{9}{|l|}{ Tumor grade } \\
\hline $\mathrm{GI} / 2$ vs $\mathrm{G} 3$ & $3.009(2.273-3.983)$ & $<0.001$ & $2.042(1.52-2.744)$ & $<0.001$ & $2.903(2.236-3.770)$ & $<0.001$ & $2.083(1.577-2.753)$ & $<0.001$ \\
\hline \multicolumn{9}{|l|}{ HER2 status } \\
\hline$+v s-$ & $1.012(0.782-1.309)$ & 0.930 & $0.853(0.647-1.126)$ & 0.262 & $\mathrm{I} .093(0.866-\mid .38 \mathrm{I})$ & 0.453 & I. $103(0.862-1.412)$ & 0.436 \\
\hline \multicolumn{9}{|l|}{ NLR } \\
\hline$<3$ vs $\geq 3$ & 3.091 (2.354-4.059) & $<0.001$ & $2.334(1.7 \mid 2-3.182)$ & $<0.001$ & $2.369(1.852-3.030)$ & $<0.001$ & $\mathrm{I} .888(\mathrm{I} .42 \mathrm{I}-2.508)$ & $<0.001$ \\
\hline \multicolumn{9}{|l|}{ PLR } \\
\hline$<147$ vs $\geq 147$ & $2.066(1.580-2.702)$ & $<0.001$ & I.302 (0.947-I.789) & 0.104 & $1.728(\mathrm{I} .36 \mathrm{I}-2.194)$ & $<0.001$ & I.I $82(0.900-1.154)$ & 0.229 \\
\hline
\end{tabular}

Abbreviations: OS, overall survival; DFS, disease-free survival; HR-, hormone-receptor negative; HR, hazard ratio; Cl, confidence interval; HER2, human epidermal growth factor receptor 2; +, positive; -, negative; NLR, neutrophil-to-lymphocyte ratio; PLR, platelet-to-lymphocyte ratio.

its significance for DFS. In the univariable analysis, both increased NLR and PLR indicated poor OS and DFS. For HER2+ patients, increased NLR was represented as an independent prognostic factor for both OS and DFS, but PLR was not (Table 3). The result of the univariable analysis suggested that elevated NLR and PLR were associated with poor OS and DFS.

\section{Discussion}

Inflammation plays a positive role in tumor initiation, occurrence, development, and progression. ${ }^{23-25}$ Neutrophils, as inflammatory and immune parameter, were recognized to be associated with tumor proliferation and metastasis by the release of inflammatory mediators, such as interleukin-8, neutrophil elastase, and matrix metalloproteinase- $9 .{ }^{26-29}$ Besides, platelets could not only promote tumor angiogenesis and metastases but also prevent tumor cells from the antitumor immune response by shielding them. ${ }^{30,31}$ Conversely, tumor-infiltrating lymphocytes could prevent the growth and spread of tumors by their antitumor immune response and are associated with better survival in patients with cancer. ${ }^{32,33}$ However, in some studies, the presence of several kinds of infiltrating lymphocytes, such as regulatory $T$ cells and programmed death-1 positive lymphocytes, is associated with worse survival..$^{34,35}$

Previously, as a combination of neutrophil/platelet and lymphocyte, NLR and PLR were proposed to be predictive markers in various solid tumors, including nasopharyngeal,

Table 3 Association between NLR/PLR and OS/DFS in different breast cancer subtypes

\begin{tabular}{|c|c|c|c|c|c|c|c|c|}
\hline \multirow[t]{3}{*}{ Variables } & \multicolumn{4}{|l|}{ OS } & \multicolumn{4}{|l|}{ DFS } \\
\hline & \multicolumn{2}{|l|}{ Univariate analysis } & \multicolumn{2}{|c|}{ Multivariate analysis } & \multicolumn{2}{|l|}{ Univariate analysis } & \multicolumn{2}{|c|}{ Multivariate analysis } \\
\hline & HR (95\% Cl) & $P$-value & HR (95\% Cl) & $P$-value & HR (95\% Cl) & $P$-value & HR (95\% Cl) & $P$-value \\
\hline \multicolumn{9}{|l|}{ HER 2+ breast cancer } \\
\hline \multicolumn{9}{|l|}{ NLR } \\
\hline$<3$ vs $\geq 3$ & $2.390(1.633-3.647)$ & $<0.001$ & $2.210(1.373-3.557)$ & 0.001 & $2.159(1.522-3.065)$ & $<0.001$ & $2.113(1.362-3.278)$ & 0.001 \\
\hline \multicolumn{9}{|l|}{ PLR } \\
\hline$<147$ vs $\geq 147$ & $1.733(1.173-2.560)$ & 0.006 & $0.953(0.577-1.574)$ & 0.852 & $1.720(1.212-2.442)$ & 0.002 & $0.911(0.582-1.424)$ & 0.682 \\
\hline \multicolumn{9}{|l|}{ TNBC } \\
\hline \multicolumn{9}{|l|}{ NLR } \\
\hline$<3$ vs $\geq 3$ & $4.055(2.733-6.015)$ & $<0.001$ & $2.546(1.627-3.984)$ & $<0.001$ & 2.585 (1.829-3.654) & $<0.001$ & $1.916(1.294-2.838)$ & 0.001 \\
\hline \multicolumn{9}{|l|}{ PLR } \\
\hline$<147$ vs $\geq 147$ & $2.517(1.725-3.672)$ & $<0.001$ & 1.709 (1.130-2.585) & 0.011 & $\mathrm{I} .727(\mathrm{I} .238-2.4 \mathrm{II})$ & 0.001 & $1.396(0.972-2.006)$ & 0.071 \\
\hline
\end{tabular}

Abbreviations: NLR, neutrophil-to-lymphocyte ratio; PLR, platelet-to-lymphocyte ratio; OS, overall survival; DFS, disease-free survival; $\mathrm{HR}$, hazard ratio; Cl, confidence interval; HER2+, human epidermal growth factor receptor 2 positive; TNBC, triple-negative breast cancer. 
pleural, lung, breast, gastric, hepatocellular, pancreatic, colorectal, prostate, endometrial, and ovarian cancers. ${ }^{8,11-13,36-46}$ However, the underlying mechanisms related to NLR/PLR and cancer are unclear. The main hypothesis is that elevated NLR and PLR resulted from increased neutrophils and platelets and/or decreased lymphocytes, which may suggest tumor progression and poor survival. Four recent meta-analysis studies confirmed the association between elevated NLR and poor prognosis in gastric, colorectal, and non-smallcell lung cancers. ${ }^{9,12,15,39}$ For breast cancer, studies about the predictive value of NLR and PLR were rare, especially for HR- breast cancer. ${ }^{8,17,19-22}$

In the present study, we validated the utility of high NLR for HR- breast cancer. Elevated PLR was significantly associated with poor OS and DFS, but this association did not remain in the multivariable analysis adjusted for age, tumor stage, lymph node involvement, tumor grade, HER2 status, and NLR. The subanalysis showed the same result in HER2+ breast cancer. However, increased NLR and PLR were both independently associated with high mortality of TNBC.

To date, there are sparse data directly investigating the usefulness of elevated NLR/PLR in HER2+ breast cancer and TNBC. Different cutoff values of NLR (3.0, 4.0, and 5.0) and PLR (185 and 292) were used in different studies. Considering the differences between human races, we used ROC analysis to find out the optimal cutoff values for this study population. As a result, the cutoff value of NLR (3.0) was consistent with that of most of previous studies. Meanwhile, the PLR value of 147 was lower than that of most of published studies, but higher than that of a research from the People's Republic of China. ${ }^{19}$ These results may contribute to differences in race and study population.

In this study, we provided additional powerful evidence for the utility of NLR in HR- breast cancer, which is consistent with most of the published studies in spite of the cutoff value..$^{8,17,19}$ However, two studies did not detect a significant association between elevated NLR and both DFS and OS in HR- breast cancer patients, which may account for small sample size. ${ }^{21,22}$ For the predictive value of PLR, only two studies performed Cox proportional hazards model in HRbreast cancer. In TNBC, the most recent study revealed that it is statistically significant between groups of high PLR $(>185)$ and low PLR $(\leq 185)$ in the multivariable analysis, but the HR was $1.27(0.76-2.14) .{ }^{8}$ Another study validated the association of increased PLR $(\geq 292)$ and clinical outcomes, but it did not remain in the multivariable analysis. ${ }^{20} \mathrm{In}$ the present study, we found that it is independently associated with patients' survival of TNBC. In HER2+ breast cancer, our result is in agreement with the most recent study with similar HR that high PLR was not independently correlated with decreased survival. ${ }^{8}$

To the best of our knowledge, this is the largest research aimed to study the utility of NLR/PLR in Chinese HR- breast cancer patients. In addition, accurate measurement of ER, PR, and HER2 status and detailed clinicopathologic parameters and treatment information are also major strengths of our study.

Nevertheless, there are some limitations in our study. First, a selection bias of retrospective, single-center study was unavoidable, and we lacked external validation. Second, substandard and delayed treatment for patients might influence patients' survival. Third, although we excluded patients with diseases that influenced NLR and PLR, there might still be patients with these diseases but not recorded in the electronic medical records. Besides, some factors that may impact the value of NLR and PLR were not recognized because of limited studies. In spite of these limitations, our study provides a robust evidence for the evaluation of the NLR/PLR effect on HR- breast cancer patients.

\section{Conclusion}

Pretreatment NLR and PLR are useful biomarkers for HRbreast cancer patients. As convenient, inexpensive, and effective biomarkers, NLR and PLR may be added to existing prognostic factors to determine treatment options in the future. Prospective investigations are needed to confirm our findings and investigate the optimal cutoff value for different races.

\section{Disclosure}

The authors report no conflicts of interest in this work.

\section{References}

1. Chen W, Zheng R, Baade PD, et al. Cancer statistics in China, 2015. CA Cancer J Clin. 2016;66(2):115-132.

2. Fan L, Strasser-Weippl K, Li JJ, et al. Breast cancer in China. Lancet Oncol. 2014;15(7):e279-e289.

3. Kohler BA, Sherman RL, Howlader N, et al. Annual report to the nation on the status of cancer, 1975-2011, featuring incidence of breast cancer subtypes by race/ethnicity, poverty, and state. J Natl Cancer Inst. 2015;107(6): djv048.

4. Kraeima J, Siesling S, Vliegen IM, Klaase JM, IJzerman MJ. Individual risk profiling for breast cancer recurrence: towards tailored follow-up schemes. Br J Cancer. 2013;109(4):866-871.

5. Sparano JA, Gray RJ, Makower DF, et al. Prospective validation of a 21-gene expression assay in breast cancer. N Engl J Med. 2015;373(21): 2005-2014.

6. Kuniyoshi RK, Gehrke Fde S, Alves BC, et al. Gene profiling and circulating tumor cells as biomarker to prognostic of patients with locoregional breast cancer. Tumour Biol. 2015;36(10):8075-8083.

7. Ali HR, Provenzano E, Dawson SJ, et al. Association between CD8+ T-cell infiltration and breast cancer survival in 12,439 patients. Ann Oncol. 2014;25(8):1536-1543.

8. Koh CH, Bhoo-Pathy $\mathrm{N}, \mathrm{Ng} \mathrm{KL}$, et al. Utility of pre-treatment neutrophillymphocyte ratio and platelet-lymphocyte ratio as prognostic factors in breast cancer. Br J Cancer. 2015;113(1):150-158. 
9. Templeton AJ, McNamara MG, Seruga B, et al. Prognostic role of neutrophil-to-lymphocyte ratio in solid tumors: a systematic review and meta-analysis. J Natl Cancer Inst. 2014;106(6):dju124.

10. Roxburgh CS, McMillan DC. Role of systemic inflammatory response in predicting survival in patients with primary operable cancer. Future Oncol. 2010;6(1):149-163.

11. Jia J, Zheng X, Chen Y, et al. Stage-dependent changes of preoperative neutrophil to lymphocyte ratio and platelet to lymphocyte ratio in colorectal cancer. Tumour Biol. 2015;36(12):9319-9325.

12. Li MX, Liu XM, Zhang XF, et al. Prognostic role of neutrophil-tolymphocyte ratio in colorectal cancer: a systematic review and metaanalysis. Int J Cancer. 2014;134(10):2403-2413.

13. Stotz M, Gerger A, Eisner F, et al. Increased neutrophil-lymphocyte ratio is a poor prognostic factor in patients with primary operable and inoperable pancreatic cancer. Br J Cancer. 2013;109(2):416-421.

14. Ishizuka M, Nagata H, Takagi K, Iwasaki Y, Kubota K. Combination of platelet count and neutrophil to lymphocyte ratio is a useful predictor of postoperative survival in patients with colorectal cancer. Br J Cancer. 2013;109(2):401-407.

15. Peng B, Wang YH, Liu YM, Ma LX. Prognostic significance of the neutrophil to lymphocyte ratio in patients with non-small cell lung cancer: a systemic review and meta-analysis. Int J Clin Exp Med. 2015;8(3): 3098-3106.

16. Kim EY, Lee JW, Yoo HM, Park CH, Song KY. The platelet-tolymphocyte ratio versus neutrophil-to-lymphocyte ratio: which is better as a prognostic factor in gastric cancer? Ann Surg Oncol. 2015;22(13): 4363-4370.

17. Pistelli M, De Lisa M, Ballatore Z, et al. Pre-treatment neutrophil to lymphocyte ratio may be a useful tool in predicting survival in early triple negative breast cancer patients. BMC Cancer. 2015;15:195.

18. Dirican A, Kucukzeybek BB, Alacacioglu A, et al. Do the derived neutrophil to lymphocyte ratio and the neutrophil to lymphocyte ratio predict prognosis in breast cancer? Int J Clin Oncol. 2015;20(1):70-81.

19. Yao M, Liu Y, Jin H, et al. Prognostic value of preoperative inflammatory markers in Chinese patients with breast cancer. Onco Targets Ther. 2014;7:1743-1752.

20. Krenn-Pilko S, Langsenlehner U, Thurner EM, et al. The elevated preoperative platelet-to-lymphocyte ratio predicts poor prognosis in breast cancer patients. Br J Cancer. 2014;110(10):2524-2530.

21. Noh H, Eomm M, Han A. Usefulness of pretreatment neutrophil to lymphocyte ratio in predicting disease-specific survival in breast cancer patients. J Breast Cancer. 2013;16(1):55-59.

22. Krenn-Pilko S, Langsenlehner U, Stojakovic T, et al. The elevated preoperative derived neutrophil-to-lymphocyte ratio predicts poor clinical outcome in breast cancer patients. Tumour Biol. 2016;37(1):361-368.

23. Grivennikov SI, Greten FR, Karin M. Immunity, inflammation, and cancer. Cell. 2010;140(6):883-899.

24. Mantovani A, Allavena P, Sica A, Balkwill F. Cancer-related inflammation. Nature. 2008;454(7203):436-444

25. Hanahan D, Weinberg RA. Hallmarks of cancer: the next generation. Cell. 2011;144(5):646-674.

26. Li XF, Chen DP, Ouyang FZ, et al. Increased autophagy sustains the survival and pro-tumourigenic effects of neutrophils in human hepatocellular carcinoma. J Hepatol. 2015;62(1):131-139.

27. Hattar K, Franz K, Ludwig M, et al. Interactions between neutrophils and non-small cell lung cancer cells: enhancement of tumor proliferation and inflammatory mediator synthesis. Cancer Immunol Immunother. 2014;63(12):1297-1306.

28. Houghton AM, Rzymkiewicz DM, Ji H, et al. Neutrophil elastasemediated degradation of IRS-1 accelerates lung tumor growth. Nat Med. 2010;16(2):219-223.
29. De Larco JE, Wuertz BR, Furcht LT. The potential role of neutrophils in promoting the metastatic phenotype of tumors releasing interleukin-8. Clin Cancer Res. 2004;10(15):4895-4900.

30. Bambace NM, Holmes CE. The platelet contribution to cancer progression. J Thromb Haemost. 2011;9(2):237-249.

31. Gay LJ, Felding-Habermann B. Contribution of platelets to tumour metastasis. Nat Rev Cancer. 2011;11(2):123-134.

32. Adams S, Gray RJ, Demaria S, et al. Prognostic value of tumor-infiltrating lymphocytes in triple-negative breast cancers from two phase III randomized adjuvant breast cancer trials: ECOG 2197 and ECOG 1199. J Clin Oncol. 2014;32(27):2959-2966.

33. Shankaran V, Ikeda H, Bruce AT, et al. IFNgamma and lymphocytes prevent primary tumour development and shape tumour immunogenicity. Nature. 2001;410(6832):1107-1111.

34. Muenst S, Soysal SD, Gao F, Obermann EC, Oertli D, Gillanders WE. The presence of programmed death 1 (PD-1)-positive tumor-infiltrating lymphocytes is associated with poor prognosis in human breast cancer. Breast Cancer Res Treat. 2013;139(3):667-676.

35. Tao H, Mimura Y, Aoe K, et al. Prognostic potential of FOXP3 expression in non-small cell lung cancer cells combined with tumor-infiltrating regulatory T cells. Lung Cancer. 2012;75(1):95-101.

36. Li X, Chen ZH, Xing YF, et al. Platelet-to-lymphocyte ratio acts as a prognostic factor for patients with advanced hepatocellular carcinoma. Tumour Biol. 2015;36(4):2263-2269.

37. Zhang WW, Liu KJ, Hu GL, Liang WJ. Preoperative platelet/lymphocyte ratio is a superior prognostic factor compared to other systemic inflammatory response markers in ovarian cancer patients. Tumour Biol. 2015;36(11):8831-8837.

38. Yamagishi T, Fujimoto N, Nishi H, et al. Prognostic significance of the lymphocyte-to-monocyte ratio in patients with malignant pleural mesothelioma. Lung Cancer. 2015;90(1):111-117.

39. Xin-Ji Z, Yong-Gang L, Xiao-Jun S, Xiao-Wu C, Dong Z, Da-Jian Z. The prognostic role of neutrophils to lymphocytes ratio and platelet count in gastric cancer: a meta-analysis. Int J Surg. 2015;21:84-91.

40. Lorente D, Mateo J, Templeton AJ, et al. Baseline neutrophil-lymphocyte ratio (NLR) is associated with survival and response to treatment with second-line chemotherapy for advanced prostate cancer independent of baseline steroid use. Ann Oncol. 2015;26(4):750-755.

41. Jiang R, Zou X, Hu W, et al. The elevated pretreatment platelet-tolymphocyte ratio predicts poor outcome in nasopharyngeal carcinoma patients. Tumour Biol. 2015;36(10):7775-7787.

42. Inoue D, Ozaka M, Matsuyama M, et al. Prognostic value of neutrophillymphocyte ratio and level of $\mathrm{C}$-reactive protein in a large cohort of pancreatic cancer patients: a retrospective study in a single institute in Japan. Jpn J Clin Oncol. 2015;45(1):61-66.

43. Cummings M, Merone L, Keeble C, et al. Preoperative neutrophil: lymphocyte and platelet:lymphocyte ratios predict endometrial cancer survival. Br J Cancer. 2015;113(2):311-320.

44. Pinato DJ, Shiner RJ, Seckl MJ, Stebbing J, Sharma R, Mauri FA. Prognostic performance of inflammation-based prognostic indices in primary operable non-small cell lung cancer. Br J Cancer. 2014;110(8): 1930-1935.

45. Kang MH, Go SI, Song HN, et al. The prognostic impact of the neutrophil-to-lymphocyte ratio in patients with small-cell lung cancer. Br J Cancer. 2014;111(3):452-460.

46. Kinoshita A, Onoda H, Imai N, et al. Comparison of the prognostic value of inflammation-based prognostic scores in patients with hepatocellular carcinoma. Br J Cancer. 2012;107(6):988-993. 


\section{Publish your work in this journal}

OncoTargets and Therapy is an international, peer-reviewed, open access journal focusing on the pathological basis of all cancers, potential targets for therapy and treatment protocols employed to improve the management of cancer patients. The journal also focuses on the impact of management programs and new therapeutic agents and protocols on

patient perspectives such as quality of life, adherence and satisfaction. The manuscript management system is completely online and includes a very quick and fair peer-review system, which is all easy to use. Visit http://www.dovepress.com/testimonials.php to read real quotes from published authors.

Submit your manuscript here: http://www.dovepress.com/oncotargets-and-therapy-journal 